\title{
Blunt scissors stromal dissection technique for deep anterior lamellar keratoplasty
}

\author{
This article was published in the following Dove Press journal: \\ Clinical Ophthalmology \\ 15 September 2014 \\ Number of times this article has been viewed
}

\section{Didar S Anwar \\ Matthew M Kruger \\ VVinod Mootha}

Department of Ophthalmology, University of Texas Southwestern Medical Center, Dallas, TX, USA
Correspondence: $\mathrm{V}$ Vinod Mootha Department of Ophthalmology, University of Texas Southwestern Medical Center, 5323 Harry Hines Boulevard, Dallas, TX 75390-9057, USA $\mathrm{Tel}+\mathrm{I} 2$ |4 6482387

Email vinod.mootha@utsouthwestern.edu
Objective: We describe a modified technique for performing lamellar dissection in deep anterior lamellar keratoplasty after failure to achieve a "big bubble" detachment of Descemet's membrane (DM) with deep intrastromal pneumatic injection.

Methods: The technique utilizes blunt lamellar dissection with blunt-tipped corneal mini scissors as an alternative to a crescent blade, which can be difficult for surgeons to master and is associated with a high risk of perforation.

Results: Other techniques of blunt dissection, such as the Melles technique, cannot be utilized after failure to achieve a big bubble, as emphysema in the stroma prevents visualization of the spatula. In contrast, our blunt scissors lamellar dissection technique takes advantage of the emphysema and microdetachments of DM created by the pneumatic injection.

Conclusion: This technique provides deep anterior lamellar keratoplasty surgeons with a simple, alternative method of baring DM or achieving a pre-DM plane with minimal residual stroma, after failure to achieve a big bubble.

Keywords: scissors, blunt, dissection, big bubble, DALK

\section{Introduction}

Anwar et al's" "big bubble" deep anterior lamellar keratoplasty (DALK) has become the treatment of choice for keratoconus, scar, and other stromal pathology. ${ }^{2,3}$ However, it is a difficult technique to master because of difficulty of achieving the big bubble. The likelihood of achieving a big bubble is not $100 \%$, even in the hands of experienced surgeons. A wide range of failure rates in achieving a big bubble has been reported, including a $14 \%$ big bubble failure rate reported by Sarnicola and Toro. ${ }^{4}$ Other surgeons have reported even higher failure rates. ${ }^{5}$ Having a backup technique after failing to achieve a big bubble can increase the DALK success rate. We herein describe a simple and effective method to aid in corneal stromal dissection for DALK after failure to achieve a big bubble.

\section{Case I}

A 50-year-old male with a history of corneal scar due to chemical burns with partial stem cell deficiency underwent DALK in the left eye (Figure 1). His preoperative best-corrected visual acuity was 20/200. Pneumatic injections failed to achieve a big bubble. The scissors DALK technique was used to effectively bare the Descemet's membrane (DM) and successfully complete the surgery (Figure $2 \mathrm{~A}-\mathrm{F}$ ). His postoperative slit lamp examination revealed an interface appearance very similar to that achieved with a successful big bubble DALK (Figure 3). Visante ocular coherence tomography (Carl Zeiss Meditec AG, Jena, Germany) revealed no apparent residual stroma over the DM (Figure 4). His postoperative best-corrected visual acuity was 20/60 at 1 month 


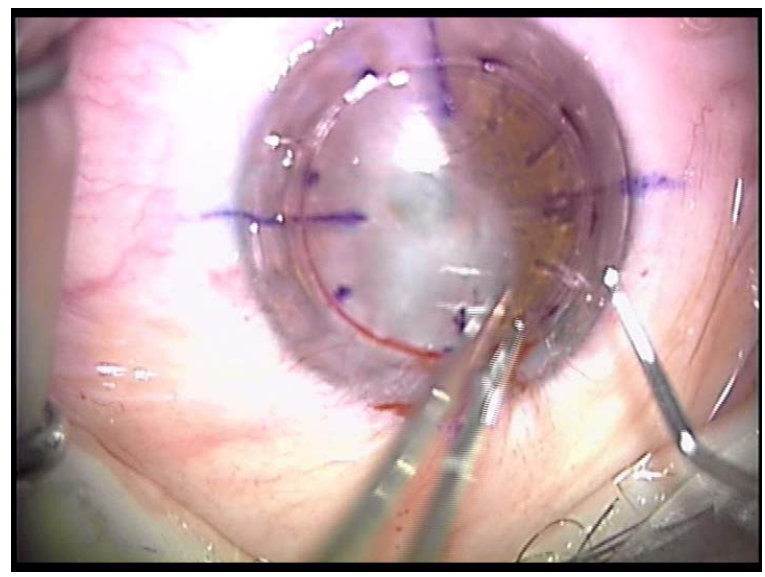

Figure I Preoperative appearance of the cornea, which shows diffuse scarring.

postoperatively. He had slow but eventually complete reepithelialization due to partial stem cell deficiency.

\section{Case 2}

A 17-year-old male presented with keratoconus with central scarring with a best-corrected visual acuity counting fingers at 3 feet. The central cornea was $<200$ microns. A big bubble was not achieved by deep intrastromal air injection. Complete stromal emphysema was noted after several attempts. The

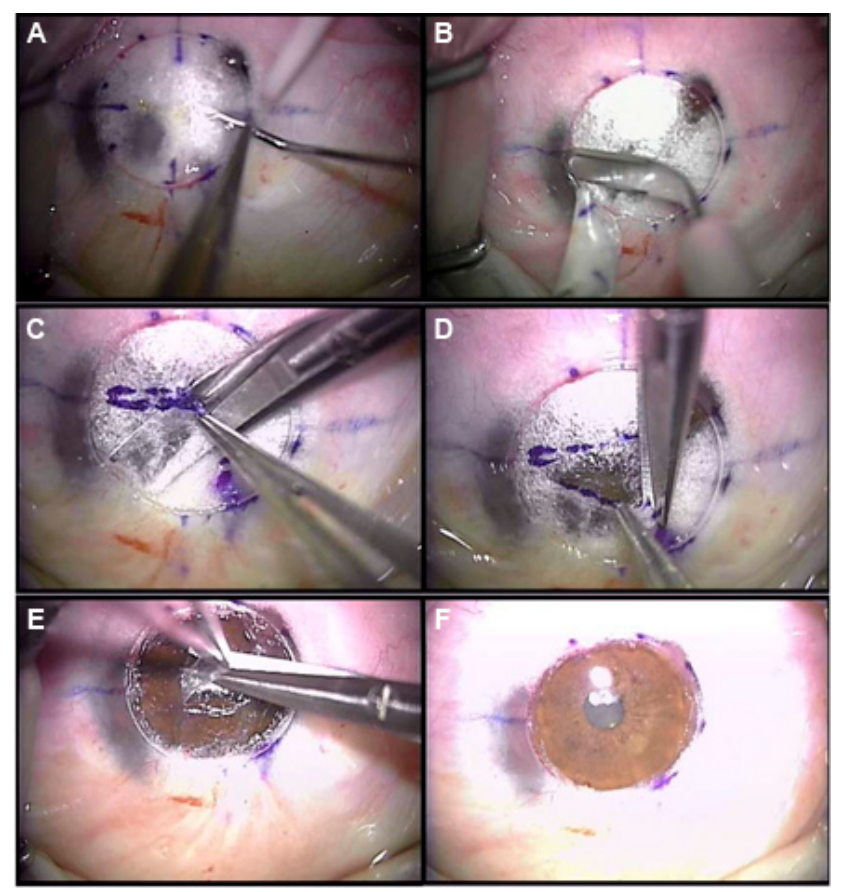

Figure 2 (A) Intraoperative photo showing emphysema of the stroma after a failed big bubble attempt. (B) A crescent blade is used to debulk the anterior $50 \%$ of the emphysematous cornea. (C) A lamellar plane is created in a quadrant of the stroma with blunt scissors dissection. (D) The dissected lamella of the stroma is then excised. (E) A similar blunt scissors dissection is carried out when pre-Descemet's stroma is reached. (F) Bare Descemet's membrane is reached.

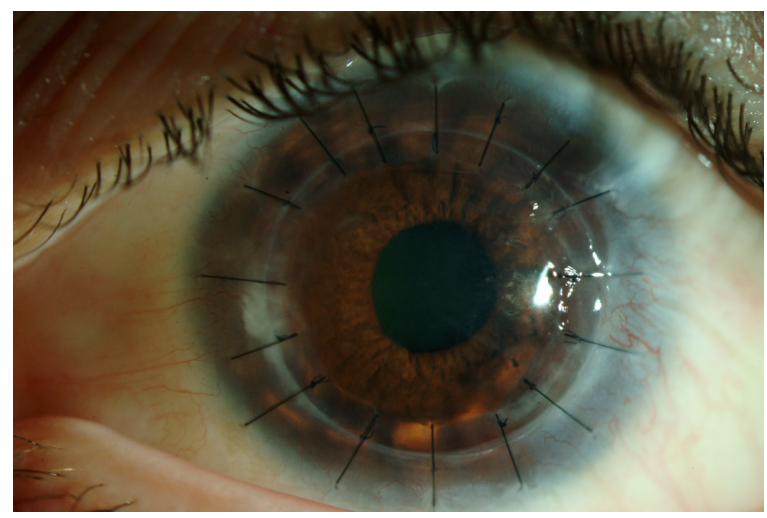

Figure 3 Postoperative slit lamp appearance.

scissors DALK technique was performed down to the level of DM. The patient's uncorrected visual acuity was 20/40 at 1 month postoperatively.

\section{Surgical technique}

This technique can be used after failure to create a big bubble in DALK (Figure 2A and Supplementary video). A crescent blade is used to debulk the anterior emphysematous stroma (Figure 2B). Using toothed forceps, such as 0.12 forceps, a piece of the stroma is grasped and is pulled up. A pair of blunt corneal mini scissors, such as blunt-tipped Castroviejo mini scissors (Storz E3218 MM, Lake Forest, CA, USA), is used to create a lamellar plane. The closed blunt tips of the scissors are gently pushed into the emphysematous stroma that developed from the intrastromal air injection during the big bubble attempt. The closed tips are then opened horizontally to create a lamellar plane. This utilizes the force of the blunt outer blade edges of the scissors for dissection. Once a lamellar plane is created, this maneuver (advancing the scissors' closed tips gently and opening them horizontally) is repeated. In this way, the lamellar plane is advanced to the periphery of the trephined cornea (Figure 2C). The anterior layers of dissected stromal lamella are excised by making radial cuts from the center to the periphery until the trephination margin is reached, thereby exposing a quadrant of dissected stromal lamella (Figure 2D). The remaining quadrants are dissected in a similar fashion and then excised along the trephination margin with scissors. The dissection is continued in a similar fashion until the most posterior stroma is visible.

At a pre-DM lamellar plane, microdetachments of DM can be visualized as shallow microbubbles. Blunt dissection continues until the DM plane is reached, taking advantage of these microdetachments. The most posterior stroma is thin and flimsy and can be grasped carefully with good 0.12 forceps while the blunt scissors dissection is continued 


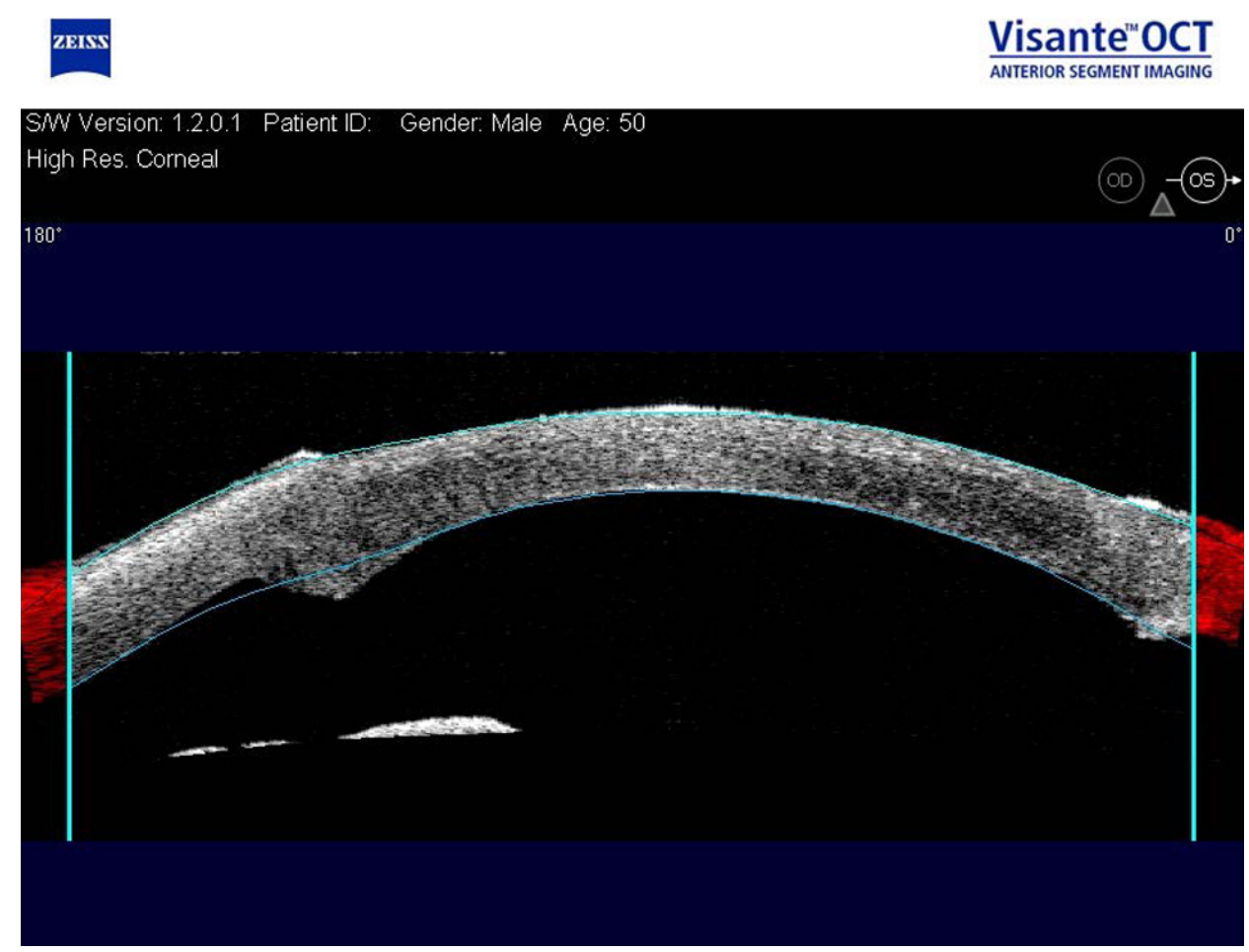

Figure 4 Visante optical coherence tomography shows no residual stroma in the interface. The bumps are the area of the attachment of the donor cornea to the recipient cornea.

(Figure 2E). Here, the closed blunt tips of the scissors are again introduced in the plane between the most posterior stroma and DM, then gently opened to bluntly dissect any remaining adhesions between the DM and pre-DM stroma. This is again carried out to the trephination margin. The dissected pre-DM stroma is again excised in quadrants. $\mathrm{DM}$ is now bared with minimal visible overlying stroma (Figure 2F). Finally, DM is stripped off the donor cornea and the cornea is sutured in place, as with any routine DALK surgery.

A modification of this technique is the stromal spiral method. The closed tips are repeatedly introduced into the previously created lamellar dissection plane and opened with forward pressure in a clockwise or counterclockwise fashion according to the surgeon's preference. The dissection plane then spirals down like the minute hand of a clock, always staying deep to the previously dissected stroma. Once at least a 1 o'clock to 2 o'clock flap of stroma has been horizontally dissected, a vertical scissor cut at the trephination mark is performed to free the dissected stromal layer, making a mobile flap that can be continually lifted and trimmed away to enhance visualization. In this fashion, the DM is reached without starting multiple new dissection planes. The residual thin stroma is difficult to remove completely. Therefore, the effort is to remove it from the center as much as possible by grasping the thin and flimsy tissue with good 0.12 forceps while gently utilizing the blunt dissection of the scissors. Leaving more residual stroma in the periphery will not affect the visual outcome. Figure 5 shows illustrations of blunt scissors dissection using the quadrant and spiral methods (Figure 5A-D).

\section{Discussion}

Blunt dissection with scissors has been long established as a valuable surgical technique in ophthalmology. In this report, we introduce the use of blunt dissection with scissors to separate and remove lamellae in the current era of "big bubble" DALK.

A crescent blade can be used for manual DALK dissection. ${ }^{6}$ Using a crescent blade for lamellar dissection of the stroma is associated with a high risk of perforation because of the sharp nature of the crescent blade.

Melles et $\mathrm{al}^{7}$ have also previously described blunt dissection for DALK. However, their technique utilizes a Melles spatula, which requires clear stroma for visualization. The Melles technique cannot be used to complete dissection after a failed big bubble, as the stromal emphysema obscures the visualization of the spatula, which is critical in this technique. This technique can at times leave a significant amount of stroma and result in interface haze. ${ }^{7}$ 


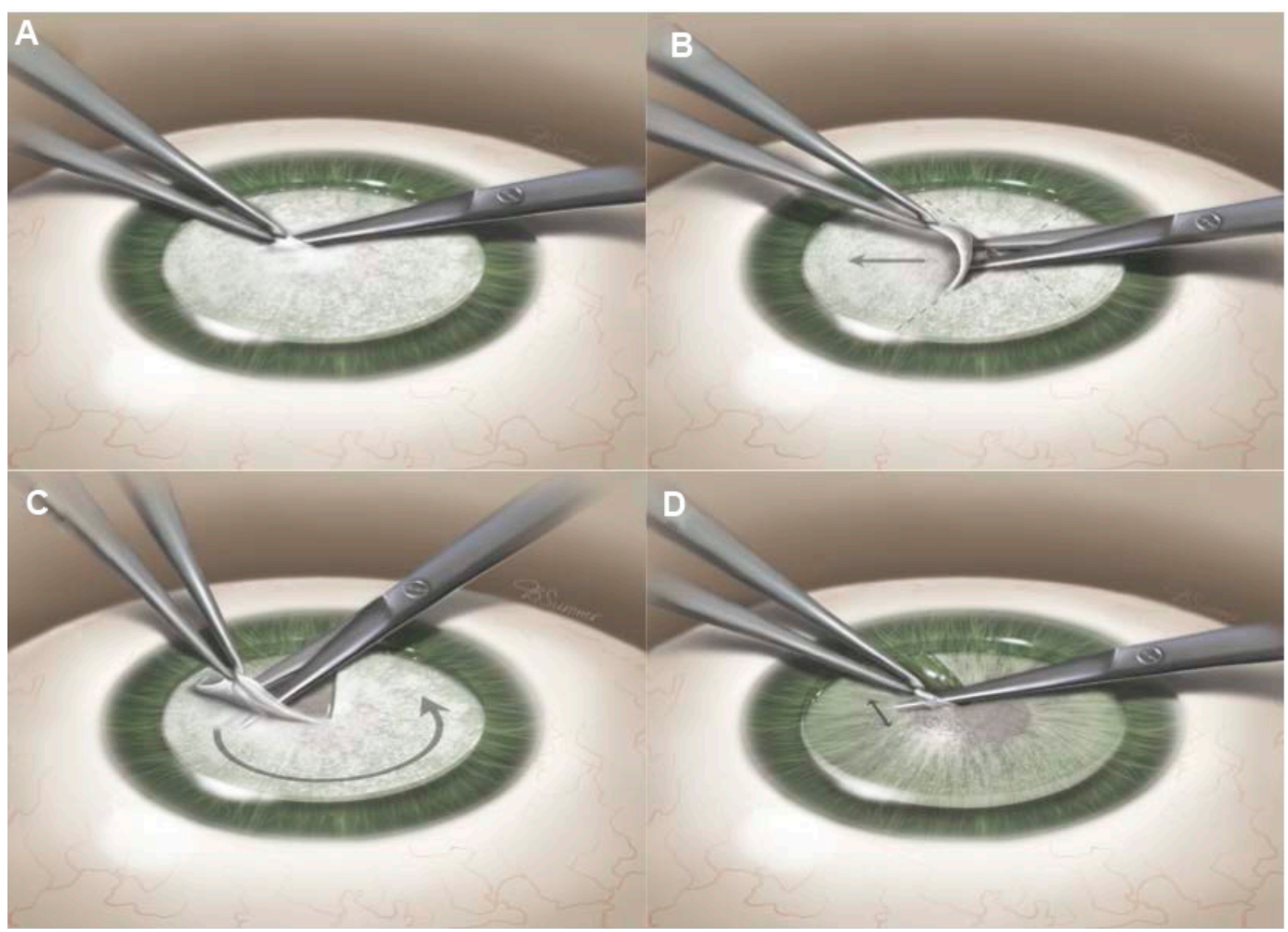

Figure 5 (A) Initiation of the lamellar plane. (B) Lamellar stromal dissection with blunt scissors using the quadrant method. (C) Lamellar stromal dissection with blunt scissors using the spiral method. (D) Blunt scissors dissection at the pre-Descemet's level.

Our technique takes advantage of intrastromal emphysema formed during attempts at a big bubble to create a lamellar plane with blunt scissor dissection. Kim et al ${ }^{8}$ have reported the creation of microdetachments at the level of DM with pneumatic injection in the absence of a big bubble. ${ }^{9}$ Lamellar blunt scissors dissection can be used to reach the level of the DM or pre-DM plane utilizing the emphysema in the stroma and microdetachments of the DM. Once the pre-DM plane is reached, the blunt scissors dissection becomes easier and the microdetachments of DM can be recognized as shallow microbubbles (Figure 6A and B). However, given the fragility of DM, dissection at this plane should be gentle and approached with caution. Therefore, overlying pre-DM stromal lamella that has been dissected should be excised frequently to minimize the force of the blunt scissor tips on the DM in a tight pocket. Since a big bubble is not formed, this technique will not dissect off the Dua's layer, as has been described recently. ${ }^{10}$ Theoretically, this should preserve the strength of the cornea.

There is risk of DM perforation with this technique. However, given the blunt nature of the dissection, the risk of perforation may be less than if proceeding with a crescent blade to perform a lamellae-by-lamellae dissection in DALK. Microperforations of DM can be managed by injecting air into the anterior chamber.

Surgeons need alternative techniques to successfully complete DALK when a pneumatic injection fails to create a big bubble. In this scenario, Sarnicola have reported the use of injection of a viscoelastic in the pre-DM plane to detach the DM from the stroma. ${ }^{11}$ Our blunt scissors stromal dissection technique also exploits the lamellar separation and microdetachments induced by the pneumatic injection. Given the blunt nature of dissection, the scissors DALK technique may be an effective method for surgeons to successfully complete DALK in the absence of a big bubble. Riss et a ${ }^{12}$ also described incising a microdetachment after a failed big bubble technique to complete DALK. However, they used blunt spatula and viscodissection to complete the DALK instead of blunt scissors dissection.

This technique is not recommended for baring the DM routinely in DALK surgery until more extensive studies show its safety in terms of risk of DM rupture. 

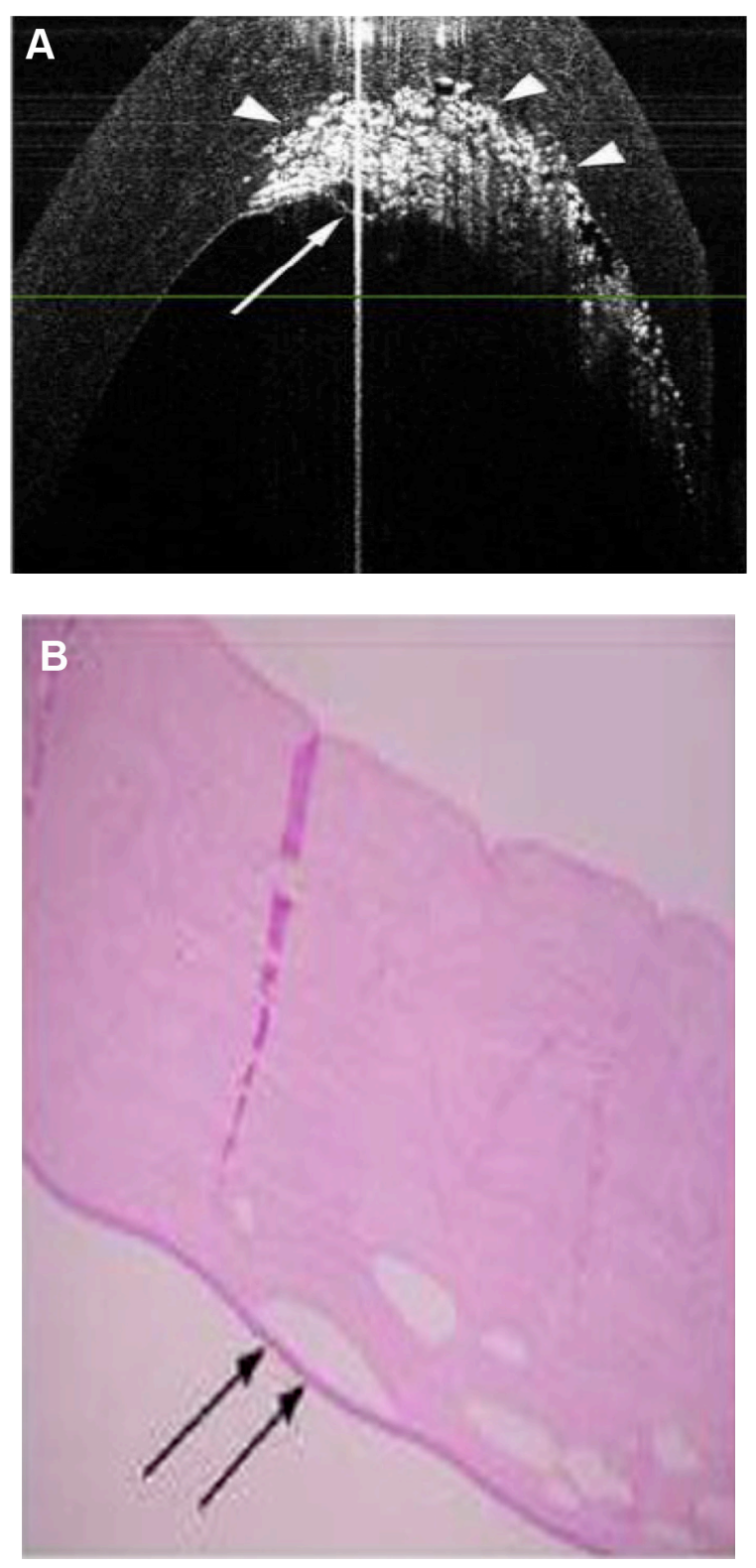

Figure 6 (A) Visante optical coherence tomography shows microdetachment. (B) Histopathological slide shows microdetachment (arrows).

Note: (A) Arrow heads show extent of deep intrastromal emphysema. The arrow points to localized detachment of Descemet's membrane.

Further studies are required to examine the visual and anatomic outcomes of the blunt scissors DALK technique.

\section{Acknowledgment}

This study was supported by an unrestricted grant from

Research to Prevent Blindness, New York.

\section{Author contributions}

All the authors have contributed their efforts to this research and manuscript preparation.

\section{Disclosure}

The authors report no conflicts of interest in this work.

\section{References}

1. Anwar M, Teichmann KD. Deep lamellar keratoplasty: surgical techniques for anterior lamellar keratoplasty with and without baring of Descemet's membrane. Cornea. 2002;21(4):374-383.

2. Fontana L, Parente G, Tassinari G. Clinical outcomes after deep anterior lamellar keratoplasty using the big-bubble technique in patients with keratoconus. Am J Ophthalmol. 2007;143;117-124.

3. Smadja D, Colin J, Krueger RR, et al. Outcomes of deep anterior lamellar keratoplasty for keratoconus: learning curve and advantages of the big bubble technique. Cornea. 2012;31(8):859-863.

4. Sarnicola V, Toro P. Blunt cannula for descemetic deep anterior lamellar keratoplasty. Cornea. 2011;30:895-898.

5. Bhatt UK, Fares U, Rahman I, Said DG, Maharajan SV, Dua HS Outcomes of deep anterior lamellar keratoplasty following successful and failed 'big bubble'. Br J Ophthalmol. 2012;96:564-569.

6. Rama P, Knutsson KA, Razzoli G, Matuska S, Viganò M, Paganoni G. Deep anterior lamellar keratoplasty using an original manual technique. Cornea. 2013;97(1):23-27.

7. Melles GR, Lander F, Rietveld FJ, Remeijer L, Beekhuis W, Binder P A new surgical technique for deep stromal, anterior lamellar keratoplasty. Br J Ophthalmol. 1999;83:327-333.

8. Kim SY, Muftouglu O, Hogan RN, et al. Histopathology and spectral domain OCT findings of pneumatic-assisted dissection in DALK. Cornea. 2012; 31(11):1288-1293.

9. Braun JM, Hofmann-Rummelt C, Schlötzer-Schrehardt U, Kruse FE, Cursiefen C. Histopathological changes after deep anterior lamellar keratoplasty using the 'big-bubble technique'. Acta Ophthalmol. 2013; 91(1):78-82.

10. Dua HS, Faraj LA, Said DG, Gray T, Lowe J. Human corneal anatomy redefined: a novel pre-Descemet's layer (Dua's layer). Ophthalmology. 2013;120(9):1778-1885.

11. Sarnicola V, Toro P, Gentile D, Hannush SB. Descemetic DALK and predescemetic DALK: outcomes in 236 cases of keratoconus. Cornea. 2010;29:53-59.

12. Riss S, Heindl LM, Bachmann BO, Kruse FE, Cursiefen C. Microbubble incision as a new rescue technique for big-bubble deep anterior lamellar keratoplasty with failed bubble formation. Cornea. 2013; 32(2):125-129. 


\section{Supplementary material}

Supplementary video This video shows the technique of using blunt scissors dissection to bare Descemet's membrane.

\section{Publish your work in this journal}

Clinical Ophthalmology is an international, peer-reviewed journal covering all subspecialties within ophthalmology. Key topics include: Optometry; Visual science; Pharmacology and drug therapy in eye diseases; Basic Sciences; Primary and Secondary eye care; Patient Safety and Quality of Care Improvements. This journal is indexed on

PubMed Central and CAS, and is the official journal of The Society of Clinical Ophthalmology (SCO). The manuscript management system is completely online and includes a very quick and fair peer-review system, which is all easy to use. Visit http://www.dovepress.com/ testimonials.php to read real quotes from published authors. 